\title{
ADDENDA FROM PRE-MENINSKI TRANSCRIPTION TEXTS TO STANISŁAW STACHOWSKI'S “OSMANLI TÜRKÇESINDE YENI FARSÇA ALINTILAR SÖZLÜĞÜ". PART VI
}

Keywords: Ottoman-Turkish, New Persian, lexical borrowing, transcription texts

\begin{abstract}
Stanisław Stachowski wrote a series of articles devoted to studies on the New Persian loanwords in Ottoman-Turkish, which were published in Folia Orientalia in the 1970 and later republished in 1998 as a single volume. Since then, however, a good number of editions of new Ottoman texts have appeared, especially transcription texts dating from before Meninski's Thesaurus (1680), which provide much new lexical material. Within this material there are many Persianisms - predictably enough where Ottoman-Turkish is concerned. This paper aims to supplement Stachowski's work with words of Persian origin taken from pre-Meninski transcription texts. It is divided into two parts, the first including data to be added to entries already recorded by Stachowski (eight articles), the second containing data that constitute new entries (three articles). A short historical-etymological note on the words dealt with also features at the end of each entry.
\end{abstract}

373. orospu (orosp1 1603); raspi ${ }^{\star}$ (?) (1584) - 1533 orospı (orospí) 'meretrice, puttana' (ArgAd. 244, ArgR. 188); ca. 1630 orospı (orospi) 'meretrix, scortum' (MontR. 156); 1584 raspenim (perhaps = rospının, gen. of rospi 'whore', assuming the tamlanan of a noun phrase is missing) 'moindre qu'vne putain' (PalPD. 554).

Der. - 1668 orospıcı (orozspigÿ) 'adulter' (IllNém. 190).

Phr. - 1672 orospı og̀l (oroßpi ogli) 'spurii' (HarsHaz. 104-105).

- < Pers. rūspī 'courtesan'. - N. 461 (XV); P. 211 (1430). 
374. oruc (1641); oruç (1533), ruç (1533), ruc* (1538), huruc (ca. 1630), huruç (ca. 1630), orunc (1650) - 1533 oruç/ruç (orúccj, rúccj) 'digiuno; quaresima' (ArgAd. 244, ArgR. 188); 1538 "fanno dui ruzi, che a dire apresso di nui due quadragesime, ogni anno, e ciascuno di questi ruzi dura un mese" (SpandSat. 239-240); 1603/1612 oruç (orutsch) ‘jejunium’ (MegThP. 1: 661; MegILT.); ca. 1630 oruç/uruç/huruc/ huruç (oruch, vruch, hurug, huruch) 'ieiunium' (MontR. 156); 1650 orunc (orung) 'digiuno' (CarrR. 262); 1668 oruç (orucs) 'quadragesima' (IllNém. 190).

Der. - 1650 oruclı/orunclı (orugzli, orungli) 'a digiuno, quadragesimale' (CarrR. 262).

Phr. - 1611 oruç et- (orutsch etmek) 'ayunar' (RJTMajd. 215) - 1603/1612 oruç tut ${ }^{*}$ ( ${ }^{\star}$ orutschutmak) ‘jejuno’ (MegThP. 1: 661, MegILT.); ca. 1630 oruç/ uruç tut- (oruch tutmak, vruch tutmak) 'ieiunare' (MontR. 156); 1668 oruç tut(orucstutarum) 'ieiuno' (IllNém. 190); 1672 uruç tut- (uruts tutmak) 'jejunium’ (HarsHaz. 198-199).

- < Pers. rūza 'fasting; fast-day'. - N. 462 (XIII); P. 212 (1332).

375. pabuç (babuç 1533); papoç (1609) - ca. 1520/1525/3o babuç (babuz [LupisON.], babucci [ITSprAd.]) 'scarpe' (LupisON. 2a; ITSprAd. 236); 1574 papuç (papuzsta [+ loc.]) 'scarpe' (VNAd. 65); 1575 babuç (babutz) 'souliers; calcei' (PostelInstr.); 1584 papuç // papuş (papuche) 'souliers' (PalBern. 321); 1587/88 papuç (paputsch) 'Schuh' (LubAd. 51); 1604 "unsere Schuech Paputsch genandt” (W. A. von Steinach: SchweickTurk. 54); 1609 "hatten aber keine Papotsch an" (M. Brandstetter: SchweickTurk. 54); 1611 babuç/pabuç (babutsch) 'çapato', (pabutsch) 'pianelle' (RJTMajd. 172, 217); ca. 1630 papuç (papuch) 'calcei, soleae exteriores' (MontR. 160); 1664 "ils appellent les souliers paboutsches [Fr. pl.]” (J. de Thévenot: ArvAdd. 27); 1668 papuç (papuch) 'sandalia' (IllNém. 191).

Der. - ca. 1520/1525/3o babuççı ( ${ }^{*}$ barbuzi [LupisON.], babutgi [ITSprAd.]) 'calzolaro' (LupisON. 1b; ITSprAd. 236); 1533 babuççı (babuccj́) 'chalzolaro, scarpettaio' (ArgAd. 143, ArgR. 43); 1574 babuç(ç)ı (babuzi, babvzi) 'calzolaro' (VNAd. 62); 1584 papuç(ç)ı (papouchi) 'courdonnier' (PalBern. 320; [ 'paponchi (PalPD. 526-527)]); 1611 babuççı (babutschi) 'çapatero' (RJTMajd. 172) - 1677 babuç(i)siz (babuccisis) 'scalzo, senza scarpe' (MascVoc. 183).

Phr. - 1650 eksill ekşi (= eski) babuc (echsi babug) 'ciabatta vechia' (sic) (CarrR. 70).

- 1641 papuc kalıbı (papugź kalibi) 'forma di scarpe' (MolDitt. 152).

- 1611 babuç çıkar- (babutsch tschikarmak) 'descalçar' (RJTMajd. 172); 1650 babucı çıkar- (babugi cicharirum) 'discalzarsi le scarpe' (CarrR. 107); 1677 papuçı/babuçı çıkar- (papuci cicarmach) 'discalzarsi', (babucci ciccaran) 'scalzatore' (MascVoc. 39, 183) - 1611 babuç giy- (babúcc ghiérum) 'calzar le scarpe' (FerrR. 87); 1677 pabuc gey- (pabuc ghieimech) 'mettersi le scarpe' (MascVoc. 95). - < Pers. $p \bar{a}-p \bar{u}$ š 'shoe, slipper'. - N. 471 (XV); P. 185 (1430).

376. paça (1680) - 1533 paça (pacciá) 'peduccio di animali' (ArgAd. 246, ArgR. 194). - < Pers. pāča 'feet (of sheep, calves, or other animals, especially when boiled), trotters'. - N. 471 (XV); P. (-). 
377. padişah (batişah 1455/56); patişah (1533), padisah (1587/88), poteşa(h)* (1584), padeşah (1608), padeşa (1611) - 1496/1501 padişah (padissach) ['sułtan, cesarz turecki'] (Constantine of Ostrovica: StachSHET. 455); [add.] 1533 patişah (patisciaah) 'jmperatore' (ArgAd. 247, ArgR. 195); 1553 "il grido loro [= of the Janissaries] è padiscià, che vuol dire - imperator padre nostro -" (B. Navagero: RelAlb.I 55); 1557 "quelli che par che vogliano chiamarlo del proprio nome lo [= the Sultan] chiamano Patisciach, che suona in lingua nostra più che imperator" (A. Erizzo: RelAlb.III 139); 1584 poteşa $(h)^{*}\left({ }^{*}\right.$ potheca $[$ recte potecha (Fr. spelling)]) 'roy' (PalPD. 526-527; [ ${ }^{\star}$ focheca (!) (PalBern. 320)]); 1587/88 "Padisah oder Sultan" (LubAd. 51); 1608 "Padeschach also nennen sie jhren Keyser /welches Wort dann heist ein erwehlten König” (SchwSt. 243); 1611 padişa/ padeşa (padisciá, padesciá) 'imperadore, re' (FerrR. 129); 1622 padişah (PadiSchach) ['Sultan'] (WennStach. 604); ca. 1630 padişah (padissah) 'imperator promiscue, uel rex potens' (MontR. 159); 1677 padişah (padisciah) 'imperadore' (MascVoc. 63).

Der. - 1650 padişalı (padisciali) 'maiestoso' (CarrR. 269).

Phr. - 1641 müstakil/müstekil padişah (mustakil/mustekil padisc=ah) 'monarca, principe che solo domina' (MolDitt. 260, 318).

- 1641 padişah çögeni (padisah ciogheni) 'scetro, bastone reale' (MolDitt. 380) - 1641 padişah elçisi (padisc=ah elcisi) 'ambasciatore del Rè' (MolDitt. 40) - 1611 padeşanun karısı (padescianún carisí) 'regina' (FerrR. 129) - 1641 padişah malı (padisc $=$ ah mali) 'ricchezza del Rè' (MolDitt. 344) - 1641 padişah mertebesi (padisc $=$ ah mertebesi) 'maestà, dignità regia' (MolDitt. 239) -1641 padişah silahleri (padisc $=$ ah szilahleri) 'arme del Rè' (MolDitt. 60) - 1641 padişah vekili (padisah vekili) 'vice Rè' (MolDitt. 480) - $\rightarrow$ 586. taht, 963. tac.

- < Pers. pād-šah 'emperor, sovereign. monarch, king'. - N. 471 (XI); P. 185 (XIII/XIV).

378. padişahi (1641) - 1677 padişahi (padisciachi) 'reale' (MascVoc. 151).

Phr. - 1677 padişahi saray (padisciachi sarai) 'palazzo dell'imperatore' (MascVoc. 120).

- < Pers. pādšāhī 'kingdom, empire, monarchy, royal dignity'. - N. (-); P. 185 (1482).

379. paha (1533); paa (XVI; 1611) - [add.] 1533 baha, paha (bachaá, pachaá) 'il costo, prezo' (ArgAd. 144, 246, ArgR. 44); 1587/88 (bunun) paha(si neder) (bu nun pahasi neder) 'wie thur ist das?' (LubAd. 18); 1611 (eyi) paha(sıdır) (ei pahasider) 'es bien pagado' (RJTMajd. 34); 1611 paa (paá) 'prezzo, ualerà; ricatto' (FerrR. 129); ca. 1630 paha (paha) 'pretium' (MontR. 159); 1650 paha (paha) 'costo, valore, valuta' (CarrR. 269); 1668 beha (behaie [+ dat.]) 'pretio' (IllNém. 268).

Der. - XVI coh (= çok) paalı (gioh paali/paale) 'è troppo cara, son troppo care' (GIITR. 1056); [add.] 1533 bahalı/pahalı (bachaalj') 'charo, pretioso, di gran prezo', (pachaalí) 'caro, cosa di prezo' (ArgAd. 144, 246, ArgR. 44, 194); 1611 bahalı (bahali, bahalhi) 'caro' (RJTMajd. 172); 1611 paalı (paalí) 'caro, di molto prezzo' 
(FerrR. 129); ca. 1630 pahalı (pahali) 'caro pretio' (MontR. 159); 1668 bahalı (bahalj) 'charum, charo pretio' (IllNém. 265, 267) - 1650 pahasuz (pahasus) 'a scrocco' (CarrR. 270); 1677 pahasuz (pahasus) 'senza costo' (MascVoc. 199).

- 1611 bahalılı (bahalelik) 'carestia' (RJTMajd. 172); ca. 1630 pahılık (pahilik) 'caritas' (MontR. 159).

- 1650 pahala- (pahalarum) 'valere di prezzo' (CarrR. 270).

Phr. - 1641 bu bahede (bubahedhe) 'a questo prezzo' (MolDitt. 56); 1677 bu pahede (bu pahede) 'a questo prezzo' (MascVoc. 16) - 1650 sehel pahaden (sehel pahaden) 'a macca, per poco o niente' (CarrR. 269) - 1611 ucuz paaya (vgiús paaiá) 'mercato, nõ caro' (FerrR. 129) - 1650 yüksek* paha (*iuchsechi paha) 'caro, prezzo alto' (CarrR. 269).

- 1641 pahası barışmak (pahasi barisc=mak) 'accordo di prezzo' (MolDitt. 11) - 1641 paha biç- (paha byzmek/bicimek) 'estimo de i beni; stimare' (MolDitt. 138, 225); 1677 baha/paha biç- (baha biccimek) 'stimo, cioè di robba', (paha bicmech) 'istimare, stimare, apprezzare’ (MascVoc. 46, 76, 231) - 1677 paha biçici (paha biccigi) 'istimatore, stimatore' (MascVoc. 76, 231) - 1650 paha et- (paha ederum) 'prezzare la mercanzia'; pahası et- (pahasi ederum) 'accordarsi di prezzo' (CarrR. 269, 270) - 1650 paha edici (paha edigi) prezzatore' (CarrR. 269) - 1650 paha ko- (paha chorum) 'costare, valere la mercanzia' (CarrR. 269) - 1611 paayle kurtar- (paáile curtarérum) 'ricattare' (FerrR. 113) - 1611 paayle kurtul- (paaile curtulúrum) 'ricattarsi' (FerrR. 113) - 1650 paha ol- (paha olmisc) 'prezzato'; pahası ol- (pahasi olurum) 'accordar il prezzo' (CarrR. 269, 270) - 1641 paha ver- (paha vermek) 'proferire' (MolDitt. 321); 1650 paha ver- (paha verirum) 'proferire, offerire' (CarrR. 269); 1677 paha ver- (paha vermek) 'proferire, cioè nella mercanzia', (paha verilmisc) 'proferito' (MascVoc. 138) - 1650 paha verici (paha verigi) 'proferente' (CarrR. 270).

- < Pers. bahā 'price, value'. - N. 472 (XII); P. 26 (1332).

380. pak (1630); pag (1630) - 1533 pak (pach) 'netto' (ArgAd. 246, ArgR. 194); ca. 1630 pak/pag (pak, pagh) 'purus' (MontR. 159).

Der. - 1611 paklık (paclíc) 'semplicità' (FerrR. 129); 1650 paklık (pachlich) 'santità' (CarrR. 270).

Phr. - 1650 paklı yer (pachli ier) 'santuario' (CarrR. 270).

- < Pers. pāk 'pure, chase, innocent; clean, neat'. - N. 472 (XI); P. 186 (1368).

383. palûze (1680); belte (1603), pelte (ca. 1630) - 1603/1612 belte (belte) 'puls' (MegThP. 2: ca. 363), 'pulmentum' (MegILT.); ca. 1630 pelte (pelte) 'pulmentum' (MontR. 160); 1650 paluze (paluze) 'polenta' (CarrR. 270).

- < Pers. pālüda 'kind of sweet beverage made of water, flour, and honey; jelly'. - N. 473 (XIV); P. 186-87 (1451).

384. pamuk (pambuk 1603); bambuk (1650) - 1533 pamuk (pamúch) 'bambagia' (ArgAd. 246, ArgR. 194); 1611 panbuk (panbuk) 'algodon' (RJTMajd. 217); 1611 pamuk (pamúc) 'bambace' (FerrR. 129); ca. 1630 pambuk (pambuk) 'gossipium’ 
(MontR. 159); 1650 bambuk (bambuq) 'bambage' (CarrR. 74); 1650 pamuk (pamuq) 'cotone' (CarrR. 270).

Der. - 1533 pamukçı (pamuchcj) 'bambagiaio' (ArgAd. 246, ArgR. 194) - 1650 bambuklı (bambuqli) 'bambagino, di bambage' (CarrR. 74).

- < Pers. pamba 'cotton'. - N. 473 (XI); P. 187 (XIV).

386. para (1603) - 1533 para (pará) 'pezo' (ArgAd. 247, ArgR. 194); 1611 para (pará) 'pezzo' (FerrR. 129); ca. 1630 para (para) 'parum' (MontR. 160); 1650 para (para)' brano, pezzo, scheggia; talento, moneta'; (parasi [+ poss.]) 'mezo, la metà' (CarrR. 271); 1677 para (para) 'pezzo' (MascVoc. 126).

Der. - 1533 paracık (paraggích) 'pezetto’ (ArgAd. 247, ArgR. 194).

- 1611 parala- (paralárum) 'lacerare’ (FerrR. 129); ca. 1630 parala- (paralanmis, paralamis) 'contritus, in frustra (sic) sectus' (MontR. 160); 1650 parala- (paralamach) 'naufragio' (CarrR. 271); 1668 parala- (paralarßün) 'rumpis'; (paralaingye) 'quousque lacerantur'; (parala[n]mistur) 'lacer' (IllNém. 191); 1677 parala- (paralamach) 'minuzzare in pezzi', (paralanmisc) 'minuzzato, fatti (sic) in pezzi' (MascVoc. 96).

Phr. - 1584 bir para (bir para) 'un peu' (PalBern. 329).

- 1650 agac parası (aghac parasi) 'fuscello, stecco' (CarrR. 271) - 1650 ciger bir parası (gigher bir parasi) 'fegatello' (CarrR. 99) - 1650 ekmek parası (echmech parasi) 'pezzo di pane' (CarrR. 271) - 1650 para kaftanı (= kaftan parasi) (para chaftani) 'gherone di veste' (CarrR. 196).

- 1650 para et- (para ederum) 'stracciare in pezzi' (CarrR. 271) - 1650 para edici (para edigi) 'stracciatore' (CarrR. 271).

- < Pers. pāra 'piece, portion'. - N. 476 (XIV); P. 187 (XIII/XIV).

388. pars (parts 1603) - 1533 pars (pars) 'pardo' (ArgAd. 247, ArgR. 195); ca. 1630 pars (pars) 'pardalis' (MontR. 160).

- < Pers. pārs 'pard; animal smaller than a leopard and trained to hunt'. According to Doerfer this Persian word is of Turkic origin (TMEN 2: 235-238). - N. 479 (IX); P. 187 (1332).

393. pazar (bazar 1533) - [The oldest Western records of this word - bazar, bazarra, bazari (pl.) in Italian (TLIO), basar in French (ArvAdd. 52) - date back to the $14^{\text {th }}$ century and are likely to have been borrowed from Persian or Arabic] - 1553 "li bazar che sono li luoghi dove si tiene la mercanzia" (Anonymous: RelAlb.I 236); 1574 pazar (pazar) 'domenica' (VNAd. 66); 1575, 1582 bazar (Polish sources: StachSHET. 64); 1587/88 bazar (basar) 'Markt' (LubAd. 37); 1603 pazar (pazaar) 'dies Solis' (MegThP. 1: 423); 1611 bazar (bazar) [without translation] (RJTMajd. 175); 1611 paza[r] (paszá[.]) 'piazza' (FerrR. 130); 1641 bazar (basar, baszar) 'fiera, mercato, il mercantare' (MolDitt. 147, 252); 1650 bazar (bazar) 'piazza da mercanti' (CarrR. 77); 1677 pazar/bazar (pasar, basar) 'domenica' (MascVoc. 287).

Der. - 1533 bazarcı (basargí) 'chi fa il mercato, trecchone' (ArgAd. 147, ArgR. 48); 1575 bazarcı ( ${ }^{*}$ bassargi) 'marchand; mercator' (PostelInstr.) - 1533 
bazarcık (basargích) 'mercatuzo' (ArgAd. 146, ArgR. 48) - 1641 bazarluk (basarluk) 'mercato, il mercantare' (MolDitt. 252); 1677 pazarluk (paszarluch) 'mercato doue si fa il mercato' (MascVoc. 93).

Phr. - 1533 maǵmun bazar (maghmún basár) 'mercato oue il compratore è offeso enormemente et puossi reuocare tale (mercato [ArgAd.]/contracto [ArgR.])' (ArgAd. 146, ArgR. 163).

- 1545 "In ogni città di Turchia v'è vna piazza la quale loro domandano Athpazàr, cioè mercato de caualli" (BassR. 60) - 1622 avrat bazar (Aurat-Basar) 'Weibermarck' (WennStach. 598); 1633 avrat bazar/bazarı (auratbazar) 'rynek dziewczy', (auratbazary) 'dziewcze rynki' (S. Twardowski: StachSHET. 38); 1654 "ouret bazari (= avret bazari), c'est à dire le marché des femmes" (du Loir: ArvAdd. 52) - 1641 baluk bazarı (baluk baszari) 'pescaria' (MolDitt. 302) - 1545 "I panni si vendano in vn'altro luogo, il quale domandano ${ }^{\star}$ Bilthpazàr [recte bitpazar], cioè mercato de Pedochi” (BassR. 62).

- 1525/30 pazar ertesi (pasarertesi) 'luni' (ITSprAd. 242; missing in LupisON.); 1533 bazar ertesi (bazár erttesí) 'lunedì' (ArgAd. 147, ArgR. 49); 1574 pazar ertes[i] (pazarhertes) 'luni' (VNAd. 66); 1584 pazar ärtesi (pazar arthesi) 'lundy' (PalBern. 324); 1587/88 bazar ertesi (basar ertesi) 'der Tagk nach dem Wochenmarkt (Montagk)' (LubAd. 37); 1603/1612 bazar ertesi/pazar ertsi (basar ertesi, pazar ertsi) 'dies Lunae' (MegThP. 1: 422; MegILT.); ca. 1630 pazar ertesi (pazar ertesi) 'lunae dies' (MontR. 160); 1641 bazar ertesi (baszar ertesi) 'lunedì' (MolDitt. 237); 1668 bazar ertesi (bazar erteszi) 'dies lunae’ (IllNém. 155); 1677 bazar/pazar ertesi (basar/pasar ertessi) 'lunedì' (MascVoc. 84, 287) - 1525/30 pazar gün* ( gur) 'domenica' (ITSprAd. 221; missing in LupisON.); 1533 bazar gün (bazár ghiún) 'domenica' (ArgAd. 147, ArgR. 49); 1584 pazar yön (pazarion) 'dimanche' (PalBern. 324); 1587/88 bazar yüni (bazar juni) 'der Tagk des Wochenmarkts (Sontagk)' (LubAd. 37); 1603/1612 bazar gün (basar giun) 'dies Solis' (MegThP. 1: 423; MegILT.); 1611 bazar güni (basar guni) 'domingo' (RJTMajd. 175); ca. 1630 pazar gün (pazar giun) 'solis dies' (MontR. 160); 1668 bazar güni (baßargiuni) 'dies Dominicus'; bazar günleri (bazargyunileri) 'nundinae' (IllNém. 155) - 1641 bazar yeri (basar ieri) 'mercato, il luogo doue si fa il mercato' (MolDitt. 252); 1677 pazar yeri (paszar ieri) 'mercato doue si fa il mercato' (MascVoc. 93).

- 1533 bazar et- (bazár edelúm (senúm ilá)) 'fareno mercato (teco)' (ArgAd. 147); 1611 mukayer pazar et- (mucchaiér pazár edérum) 'pigliar a patto' (FerrR. 130); 1650 bazar et- (basar ederum) 'mercatare' (CarrR. 77) - 1641 bazara git- (baszara gheden) 'spenditore' (MolDitt. 419) - 1650 bazar ol- (bazar olmisc) 'mercatato' (CarrR. 77).

- < Pers. bāzār 'market'. - N. 483 (XIII); P. 30-31 (1291-1312).

394. peder (1641) - 1677 peder (peder) 'padre' (MascVoc. 117).

- < Pers. padar 'father'. - N. 484 (XIV); P. (-).

395. pehlivan (pehlevan 1641); behlevan (1538), pelivan* (1548), pehelvan (1650) 1538 "li Bechlevani [It. pl.] (...) sonno luctatori grandissimi" (SpandSat. 220); 
1548 "tiene el gran Turco trenta huomini chiamati ${ }^{*}$ pelivander (recte pelivanler) (...), quali giocano alle bracce" (MenTratt. 166); 1568 "trente hommes forts $\&$ robustes, membrus \& nerueux (...), appelés par les Turcs ${ }^{\star}$ Peluiander (...), qui signifie luiteurs" (NicQLivr. 99); 1584 "le Seigneur fit aussi venir ses luicteurs, qu'ils appellent ${ }^{*}$ Pelinandres (elsewhere ${ }^{\star}$ Pelliandres)” (PalBern. 292); 1622 "Ringer / bey den Türcken ${ }^{\star}$ Pelvianders genañt" (WennStach. 604).

Der. - 1533 pehlivanluk (pekliuanlúch) 'ualentigia' (ArgAd. 247, ArgR. 196). Phr. - 1650 büyük pehelvan (buiuch peheluan) 'campione' (CarrR. 272).

- < Pers. pahlawān 'hero, champion, brave warrior, strong athletic man'. - N. 484 (XIV); P. 189 (XIII/XIV).

396. pehriz ( ${ }^{*}$ pekeris [recte peheriz] 1533); peyriz (1611), peeriz (ca. 1630) - [add.] 1533 peheriz (pekerís) 'dieta che si fa quando uno ha male abstinendosi dal mangiare et bere' (ArgAd. 247, ArgR. 196); 1611 peyriz (peirís) 'astinenza' (FerrR. 130); ca. 1630 perhiz/peeriz (perhiz, peeris) 'abstinentia' (MontR. 161); 1677 pehriz (pehris) 'astinenza, dieta' (MascVoc. 18, 38).

Phr. - ca. 1630 perhiz et- (perhis etmek) 'dietam, abstinentiã facere' (MontR. 161).

- < Pers. parhīz 'abstinence'. - N. 487 (XI); P. (1430).

398. pencere (1603); penşere/pänşere (1587/88), pincerä (1611), pencerä (1650) - 1533 pencere (pengére) 'finestra' (ArgAd. 248, ArgR. 197); 1587/88 penşere/pänşere (penschere, panschere) 'Fenster' (LubAd. 51); 1611 pencere (pengere) 'fenestra' (RJTMajd. 217); 1611 pincerä (pingierá) 'fenestra' (FerrR. 130); 1650 pencerä (pengerah) 'fenestra' (CarrR. 273); 1672 pencere (pengereler [+ pl.]) 'balconi o finestre' (MascVoc. 19).

Phr. - 1650 pencerä demiri (pengera demiri) 'ferrata, fenestra' (CarrR. 120). - < Pers. panğara 'cage; window'. - N. 485 (XIV); P. 190 (XIV).

399. pençe (1641) - 1533 panca (pangiá) 'brancha delli animali, zampa' (ArgAd. 246, ArgR. 194); 1650 pança (pancia(h)) 'artigli d'uccello; beccacenere' (CarrR. 270); 1677 pençe (pence) 'zampa, branca' (MascVoc. 278).

Phr. - ca. 1630 pank açmak $[<$ panç (a)* açmak] (pank achmak, pank acciarum) 'palmam aperire' (MontR. 159).

- < Pers. panğa 'palm of the hand with the five fingers; claws'. - N. 485 (XIV); P. 190 (XIV).

401. perçem (perçin 1668); perçe (1533) - 1533 perçe (percé) 'cioccha di capelli' (ArgAd. 248, ArgR. 197); 1650 perçem (prčm) 'zazzara, capelliera' (CarrR. 273).

Der. - 1650 perçemli (prčmly) 'zazzeroso' (CarrR. 273).

- < Pers. parčam 'lock of hair (especially waving over the forehead)'. - N. 487 (XV); P. 191 (1482).

402. perdah (1641), perdaht (1680); perdat (1533) - 1533 perdah (perdách) 'liscio de' corami' (ArgAd. 248, ArgR. 197). 
Phr. - 1533 perdah/perdat et- (perdách edérum) 'liscio choiami et simili', (sacchalí perdátt edérum) 'rado la barba' (ArgAd. 248, ArgR. 197); 1611 perda et- (perdá edérum) 'perfettionare qualche cosa, ripolire nel perfettionare' (FerrR. 130); 1650 perda/perdah et- (perda(h) ederum) 'lisciare, polire, rischiarare' (CarrR. 273); 1677 perda et- (perda etmech) 'pulire, dare il lustro, strisciare, lisciare' (MascVoc. 143, 234) - 1650 perda/perdah edici (perda(h) edigi) 'lisciatore, pulitore, rischiaratore' (CarrR. 273) - 1677 perda ol- (perda olunmisc) 'strisciato' (MascVoc. 234).

- < Pers. pardāxt 'finished, complete; polished; finish, polish'. - N. 487 (XV); P. (-).

404. perde (1603) - 1533 perde (perdé) 'cortina che si mette accioché non si uegga, parauento o parapetto che si fa alle finestre per non essere uisto' (ArgAd. 248, ArgR. 197); 1611 perde (perde) 'schleier' (RJTMajd. 217); ca. 1630 perde (perde) 'sepes' (MontR. 161); 1650 perde (perde) 'cortina di letto, parapetto' (CarrR. 273).

Phr. - 1641 döşek perdesi (doscek perdesi) 'padiglione da letto' (MolDitt. 288) - 1650 yüz perdesi (giuz perdesi) 'visiera' (CarrR. 273) - 1641 tahde perdesi (tachdhe perdesi) 'tauolato, diuisa da tauola' (MolDitt. 447).

- < Pers. parda 'veil, curtain, tapestry, caul, film, membrane'. - N. 487 (XIII); P. 191-92 (1332).

407. perestār (1680); punuste (ca. 1630) - ca. 1630 punuste (punuste) 'seruus' (MontR. 163).

- < Pers. parastār 'worshipper; servant, slave, male or female', pirista 'handmaid'. - N. (-); P. 192 (XV/XVI).

408. pergel (1603); brigel (1587/88), pirçel (1611) - 1533 pergel (perghiél) 'seste' (ArgAd. 248, ArgR. 198); 1587/88 brigel (brigel) 'Zirckel' (LubAd. 37); 1611 pirçel (pirciél) 'compasso' (FerrR. 130); ca. 1630 pergel (perguiel) 'compassus' (MontR. 161); 1650 pergâl (perghial) 'compasso' (CarrR. 273).

Phr. - 1650 pergâl kulan- (perghial chulanurum) 'compassare, adoperar il compasso', (perghial chulanmisc) 'compassato' (CarrR. 273).

- < Pers. pargār 'pair of compasses'. - N. 487 (XIII); P. 192 (2/XV).

410. peri (1709 [but the word was recorded by Meninski (L. R.)]) - 1533 peri (perí) 'gigante' (ArgAd. 248, ArgR. 198).

- < Pers. parī 'good genius, fairy'. - N. 487 (XI); P. 192 (XIII/XIV).

412. perşembe (1603); besembe // beșembe (1525/30), pesempe // peşempe (1574), pesembe (?) (1584), berşembe (1611) - 1525/3o besembe // beşembe (besembe) 'giouedì' (ITSprAd. 237; missing in LupisON.); 1533 peşembe (pescembé) 'giouedì' (ArgAd. 248, ArgR. 198); 1574 pesempe // peşempe (pesempe) 'giouedì' (VNAd. 66); $\mathbf{1 5 8 4}$ pesembe // peşembe (pesembe) 'jeudy' (PalBern.); 1587/88 peşembe (peschembe) 'der funfte Tagk (Donnerstag)' (LubAd. 51); 1611 peşembe (pescembé) 'giouedì, giorno della settim(an)a' (FerrR. 130). 
Phr. - 1611 berşembe güni (berschembe guni) '^mier./edes' [the meanings 'Wednesday' and 'Thursday' have been assigned to wrong days, see 72. çarşembe] (RJTMajd. 176); ca. 1630 peşembe gün (pescembe giun) 'Iouis dies' (MontR. 162).

- < Pers. panğ-šaṃbih ‘Thursday'. - N. 488 (XIII); P. 190 (1451).

416. perva (1641).

Der. - 1677 pervasuz (peruasus) 'alla libera' (MascVoc. 11).

- < Pers. parwā 'desire, inclination, affection; care, solicitude, anxiety, fear, terror, stupor'. - N. 489 (XV); P. 193 (XV).

417. pervane (1641) - 1677 pervane (peruanè) 'farfalla; lucciola, animaletto' (MascVoc. $48,84)$.

- < Pers. parwāna 'moth, particularly such as fly about a candle at night'. - N. 489 (XIV); P. 194 (XIII/XIV).

418. pervaz (1668) - 1533 pervaz (peruás) 'orlo, orliccio, fimbria' (ArgAd. 248, ArgR. 198); 1650 pervaz (peruas) 'cornice, frontiera, difesa' (CarrR. 273).

Phr. - $\rightarrow$ 215. ibrişim.

- < Pers. parwāz'small beam or batten for roofing a house; veil, shirt, shift, anything that covers or conceals', parwaz 'kind of border or selvedge round a garment'. - N. 489 (XIV); P. 194 (1525/26).

423. peşkeş (1641); peskeş (1533), piskeş (1611) - 1533 peskeş/peşkeş (peschiésc, pescichiésc) 'presente, dono' (ArgAd. 248, ArgR. 198); 1611 piskeş (pischésc) 'presente, dono' (FerrR. 131); 1677 peşkeş (pesc=chesc) 'dono' (MascVoc. 42).

- < Pers. pīš-kaš 'magnificent present, such as is only presented to princes, great men, superiors, or sometimes to equals'. - N. 489 (XIV); P. 200 (XIV).

424. peşkir (1603); piskir (?) (ca. 1520), peskir (?) (1525/30), besker (1587/88) - ca. 1520 piskir // pişkir (pischir) 'touaglia' (LupisON. 2a); 1525/30 peskir // peşkir (peschir) 'touaglia' (ITSprAd. 242); 1533 peskir (peschír) 'touaglia' (ArgAd. 248, ArgR. 198); 1587/88 peşkir (peschkir) 'Tischtuch, gemein Facelet, Schnuptuch', besker (bessker) 'Tischtuch' (LubAd. 51); 1611 beşkir (beschkir) 'manteles' (RJTMajd. 176).

Der. - 1672 peşkirci (peskirgsi) 'qui mensae mappulas circumducit' (HarsColl. 333; Stachowski records Nagy de Harsány’s word without giving its meaning) - 1533 peskircik (peschirgích) 'touagliolino' (ArgAd. 248, ArgR. 198); 1677 peşkircik (pesc=chirgich) 'touaglietta, saluietta' (MascVoc. 251).

- < Pers. pīš-gīr ‘towel, napkin'. - N. 489 (XV); P. (-).

425. peştahta (peştakta 1641) - 1650 peştahta (pesctachtah) 'banco da scriuere; studiolo' (CarrR. 274).

- < Pers. pī̌r-taxta 'portfolio; desk'. - N. (-); P. (-). 
426. peștimal (1672); biştemal (1611), pestämal (1611), pestemal (?) (ca. 1630), peștmal (1650), piştirmak (1650) - 1611 biştemal (bischtemal) 'manteles' (RJTMajd. 177); 1611 pestämal (pestamál) 'touaglia' (FerrR. 131); ca. 1630 peştemal // pestemal (pestemal) 'mappa' (MontR. 162); 1650 peştmal/piştirmak (pesctmal) 'grembiule', (pisctirmach) 'sciugatoio' (CarrR. 274).

- < Pers. pušt-māl 'apron'. - N. 490 (not dated); P. (-).

427. peydah (1641) - 1677 peydah (peidah) 'fauola; fresco, nuouamente fatto; inuenzione, finta; menzogna, bugia' (MascVoc. 48 passim).

Phr. - 1650 peydah et- (peidah ederum) 'inuentare', (peidah etmech) 'concetione' (CarrR. 274) - 1677 peydah edici (peidah edigi) 'ingegnero; inuentore' (MascVoc. 69, 74) - 1650 peydah ol- (peidah olmisc) 'concepito, inuentato' (CarrR. 274); 1677 peydah ol- (peidah olunmisc) 'inuentato' (MascVoc. 74).

- < Pers. paidā 'clear, evident, manifest; born, produced, discovered, invented'. - N. 490 (XIII); P. 196 (1482).

428. peygamber (begamber 1455/56); pe(h)amber/pe(h)ambar (1496/1501), be(m)ber (ca. 1520), pengaber (1548), bereber (?) (1580) - 1496/1501 pe(h)amber/pe(h)ambar (pe(h)amber, pe(h)ambar) ['prorok'] (Constantine of Ostrovica: StachSHET. 461); 1574 pegamber (pegamber) 'li santi' (VNAd. 66); 1575 pegamber 'sainct; propheta' (PostelInstr.); 1584 pegamber (pegamber) 'les saincts' (PalBern. 319); 1587/88 "Noa, dem sie den Nahmen Peigamber geben” (LubAd. 52); 1608 "begamber Prophet" (SchwSt. 250); 1611 begamber (begamber) 'propheta, propheto' (RJTMajd. 175); 1612 peygamber (peigamber) 'propheta' (MontR. 160); ca. 1630 pegamber (pegamber) 'profeta, sanctus' (MontR. 160); 1672 pegamber (pegamber) 'propheta' (HarsHaz. 54-55); 1677 pegamber (pegamber) 'profeta' (MascVoc. 138).

Der. - 1533 pegamberlük (peghamberlúch) 'profetia' (ArgAd. 247, ArgR. 196); 1677 pegamberlik (pegamberlich) 'profezia' (MascVoc. 138).

Phr. - 1548 "Propheta Mahomet, il quale per nome è detto Accvrzamam Pengaber (= Ahirzaman Peygamber), cioè Propheta vltimo” (MenTratt. 18) - 1677 dişi pegamber (disci pegamber) 'profetessa' (MascVoc. 138).

- ca. 1520/1525/3o Isi be (m)ber (esse beber) 'Christo' (LupisON. 1a; ITSprAd. 240 [bämbär]); 1545 "Issápehambèr (elsewhere Hissapechamber), cioè Giesu Christo” (BassR. 73); 1580 Isı bereber (?) (esse bereber) 'Christo' (BVenON. 1); 1608 "Isus Begamber der Prophet Jesus" (SchwSt. 235).

- 1677 pegamberlik et- (pegamberlich etmech) 'profetizare' (MascVoc.138).

- <Pers. paigām-bar 'messenger; prophet, apostle'. - N. 490 (XII); P. 196 (1291-1312).

429. peyk (1546); beyk* (1538), payk* (1636) - 1533 peyk (peích) 'corriere a pie” (ArgAd. 248, ArgR. 198); 1538 "sono alchuni adimandati Beichi [It. pl.] (...) et sonno questi corrieri dello imperatore che vanno a piedi" (SpandSat. 219); 1539 "quaranta Peych, cioè corrieri à piedi" (RambLibT. 19v); 1548 "cento Persiani chiamati 
peicler (...): questi vãno auanti la testa del cauallo del Signore a piedi senza calze \& senza scarpe (...); \& vanno di continuo ouunque il gran Turco vada (...) \& camminano notte \& giorno senza mai posarsi" (MenTratt. 155-157); 1568 "quarante Laquays, ou estafiers de nation Persienne, appelés en leur langue Turquesque Peicz, ou Peiclars" (NicQLivr. 95); 1573 "li peic (...) servono come staffieri" (C. Garzoni: RelAlb.I 418); 1584 "cinquante laquais, qu'ils appellent Peich" (PalBern. 263); 1587/88 "die Lackeien, Peickler genant" (LubAd. 51); 1598 "Peich del Gran Signore, cioè staffiero" (SorOtt. 10); 1608 "Peikler seyn Lakeyen / die Lauffen also schnell /daß sie in zweyen Tagen weiter kommen / dann einer in dreyen Tagen reuten kann" (SchwSt. 243); 1611 peyik (peíc) 'alabardiere' (FerrR. 131); 1622 "Peicken [G. pl.] Lackeyen” (WennStach. 604); 1636 payk (paików [Pol. gen. pl.]) ['służąci, lokaj, pokojowy’] (St. Oświęcim: StachSHET. 455-456); 1650 peyk (peich) 'letterario, staffieri' (CarrR. 274).

Phr. - 1668 "the Peikbashi, or Commander of that sort of pages which are called Peiks” (RycautPSt. 195); 1672 peyk başı (peikbasi) 'celerum capita, qui utrinque à latere equi Imperatoris, vestibus, auro \& argento intertextis, tàm bello, quàm pace procedunt' (HarsColl. 331).

- < Pers. payk 'running footman; carrier, messenger'. - N. 490 (XIII); P. 196 (1430).

430. peymane (1641) - 1677 peymane (peimane) 'cal[i]ce' (MascVoc. 24).

- < Pers. paimāna 'cup, goblet, bowl'. - N. 490 (XV); P. 197 (XIII/XIV).

431. peynir (peyner 1544/48); piyanir (?) (ca. 1520); penyir (1533), peynyir (1611) 1496/99 penir (penir) 'keess' (HarffSt. 136); ca. 1520 piyanir (?) (pianir) 'formazo' (LupisON. 2a); 1525/30 penir (penir) 'formazzo' (ITSprAd. 220); 1533 penyir (pegnír) 'chascio, formaggio' (ArgAd. 248, ArgR. 197); 1575 peyner (peiner) 'fourmage; caseus' (PostelInstr.); 1584 penir (penir) 'formage' (PalBern. 322); 1587/88 penir ${ }^{\star}{ }^{\star}$ pemr) 'Kese' (LubAd. 15); 1611 peynyir (peignir) 'queso' (RJTMajd. 217); ca. 1630 penyer (penier) 'caseus' (MontR.161); 1641 peynir/penir (peinir, penir) 'cascio, formagio' (MolDitt. 82, 152); 1650 peynir (peinir) 'formagio' (CarrR. 274); 1677 peynir (peinir) 'formaggio' (MascVoc. 52).

Der. - 1533 penyircik (pegnirgích) 'chasciolino' (ArgAd. 248, ArgR. 197) - 1650 penirici (penirigi) 'caciaro che lo fa [il cacio]' (CarrR. 273).

Phr. - $\rightarrow$ 290. maye, 572. şeker, 888. lor.

- < Pers. panīr 'cheese'. - N. 490 (XIV); P. (-).

433. pilâv (1544/48) - [The Italianised form pilao has not been taken into account] 1575 pilav (pilau) 'des mets de ris; oriza cocta' (PostelInstr.); 1604 "ein süess Essen Pillau genandt" (W. A. von Steinach: SchweickTurk. 58); 1640 "le potage de ris, qu'ils appellent Pillauw" (du Loir: SchweickTurk. 58); 1664 "Ce pilau est du ris qu'ils mettent dans vne marmite auec vne poule, du mouton, du boeuf, ou seulement l'vne de ces choses" (J. de Thévenot: ArvAdd. 433).

- < Pers. palāw, pilāw' dish composed of flesh or fish highly seasoned (...), covered and heaped over with rice newly boiled (...)’. - N. 492 (XIV); P. (-). 
434. pirinç (1553); brinş (1608), perinç* (1603) - 1533 pirinç (pirínc) 'riso, legume' (ArgAd. 249, ArgR. 199); 1587/88 pirinç (pirintsch) 'Reis' (LubAd. 52); 1603/1612 pirinç (pirintsch) 'oryza' (MegThP. 2: 182; MegILT.); 1608 "Brinsch Reis” (SchwSt. 227); 1611 perinç (perintsch) 'riso' (RJTMajd. 218); 1611 prinç (príncc) 'riso, legume da mãgiare' (FerrR. 131); ca. 1630 pirinç (pirincz) 'risum' (MontR. 162); 1668 pirinç (pirincs) 'oriza' (IllNém. 191); $1677 \operatorname{princ}(i)$ (pringi [+ poss.]) 'riso, legume' (MascVoc. 160).

Der. - 1677 princilik (pringilich) 'risara, luogo doue nasce il riso' (MascVoc. 160). Phr. - 1603 perinç ${ }^{*}$ çorba ( ${ }^{*}$ perinzht sorba) 'oryza' (MegThP. 2: 182).

- < Pers. birinğ 'rice’. - N. 493 (XIII); P. 199 (1/XV).

435. pirinç (pirinc 1680); prinç (1533) - 1533 prinç (prínc) 'bronzo' (ArgAd. 249, ArgR. 200). Phr. - 1677 pirinc(i) bokı (piringi bocchi) 'schiuma d'ottone' (MascVoc. 188). - < Pers. piring 'copper'. - N. 493 (not dated); P. (-).

436. piruze (peruze 1603) - 1533 peruze (perusé) 'turchina' (ArgAd. 248, ArgR. 198); 1611 piruze (pirusé) 'pietra torchina' (FerrR. 131): 1612 peruze (peruse) 'jaspis aerizusa, Türckiß’ (MegILT.); ca. 1630 peruze (peruze) 'yaspis aerizusa, turchina, eranos' (MontR. 161); 1677 peruze (perusze) 'turchina, gemma' (MascVoc. 260).

Phr. - 1533 toprak peruze (toprách perusé) 'roccha delle turchine' (ArgAd. 248, ArgR. 245).

- < Pers. pìrūza 'turquoise, kind of blue gem'. - N. (-); P. 199 (1482).

438. pişman (puşman [recte püşman] 1641); peşman (ca. 1630) - 1533 pişman (piscimán) 'pentito' (ArgAd. 249, ArgR. 199); ca. 1630 peşman (pesman) 'poenitens' (MontR. 162); 1650 püşman (puscman) 'penitente' (CarrR. 275).

Der. - 1533 pişmanluk (piscimanlúch) 'pentimento' (ArgAd. 249, ArgR. 199); 1603/1612 peşma[n]luk ( ${ }^{*}$ peschmaluk) 'poenitentia' (MegThP. 2: 286; MegILT.); ca. 1630 peşmanlık (pesmanlik) 'poenitentia' (MontR. 162); 1650 pişmanlık (piscmanlich) 'pentimento' (CarrR. 275).

Phr. - 1533 pişman et- (piscimán etterúrum) 'fo pentire' (ArgAd. 249, ArgR. 199) - 1533 pişman ol- (piscimán olúrum) 'pento' (ArgAd. 249, ArgR. 199); ca. 1630 peşman ol- (pesman olmak) 'poenitere aliquem' (MontR. 162); 1650 pişman ol- (piscman olurum) 'pentirsi', (piscman olmisc) 'pentito' (CarrR. 275).

- < Pers. pišmān 'penitent'. - N. 494 (XII); P. 200 (XIII/XIV).

440. piyade (1680) - 1533 piyada (piadá) 'fante ad pie', (piadalér [+ pl.]) 'fanteria' (ArgAd. 249, ArgR. 199).

- < Pers. piyāda 'footman, foot-soldier; infantry'. - N. 494 (XIV); P. 200 (1445).

441. piyale (1641) - 1533 piyale (pialé) 'bicchiere' (ArgAd. 249, ArgR. 199); ca. 1630 piyale (pialle) 'poculum' (MontR. 162).

Der. - 1533 piyaleci (pialeggí) 'bicchieraio' (ArgAd. 249, ArgR. 199).

- < Pers. piyāla 'cup, goblet or drinking glass'. - N. 494 (XIII); P. 201 (XIII/XIV). 
442. piyaz (1680); payaz // pryaz (1650) - 1650 payazler // pryazler (paiazler) 'complimenti di parole' (CarrR. 274).

Der. - 1650 payazıcı // pıyazıcı (paiazigi) 'compito, cerimonioso' (CarrR. 274).

- < Pers. piyāz ‘onion'. - N. 494 (XIV); P. (-).

443. postaki (1641) - 1611 posteki (postechí) 'coiro, pelle' (FerrR. 131).

- < Pers. pūstak 'Häutchen' (JunkerAlaviW. 137). According to Stachowski from pustāki 'id.', word not found in Persian dictionaries. - N. (-); P. (-).

445. pul (1544/48); ful (1575) - 1575 pul/ful (pul, ful) 'mõnoye deraĩ; numus aereus' (PostelInstr.); 1611 pul (púl) 'danaro' (FerrR. 131); ca. 1630 pul (pul) 'rotula ad ludendum' (MontR. 162).

Phr. - 1650 pul balık (= balık pulu) (pwl bālyk) 'rischa, scaglia di pesce' (CarrR. 275).

- 1650 pulı çıkar- (puli *cicharum) ‘scagliare’ (CarrR. 275).

- < Pers. pūl 'small piece of copper coin; money'. - N. 507 (XII); P. 201 (XIII/XIV).

447. puşt (1641) - 1533 puşt (pusct) 'uno che uuole le pesche' (ArgAd. 249, ArgR. 20); 1650 puşt (pusct) 'bagascione, bardassa' (CarrR. 275).

- < Pers. pušt 'the back; catamite'. - N. 508 (XIV); P. (-).

448. put (1455/56) - 1533 put (put) ‘jdolo’ (ArgAd. 249, ArgR. 20o); 1611 put (pút) 'idolo, ritratto in rilieuo' (FerrR. 131); ca. 1630 put (puth) 'idolum, immago' (MontR. 163); 1677 put (put) 'idolo, statua, tauola, pittura' (MascVoc. 61, 229).

- < Pers. but 'idol, image, any figure that is object of adoration'. See TMEN 2: 261-262. - N. 508 (X); P. 50 (XIII/XIV).

449. putperest (putperes 1641) - 1677 putperest (put perest) 'idolatra' (MascVoc. 61).

Der. - 1677 putpereslik (put pereslich) 'idolatria' (MascVoc. 61).

- < Pers. but-parast 'idolater'. - N. 508 (XIV); P. (-).

Abbreviations

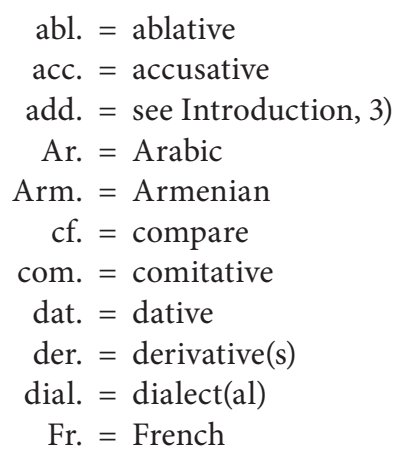

$$
\begin{aligned}
\text { Engl. } & =\text { English } \\
\text { G. } & =\text { German } \\
\text { gen. } & =\text { genitive } \\
\text { Gr. } & =\text { Greek } \\
\text { It. } & =\text { Italian } \\
\text { Lat. } & =\text { Latin } \\
\text { loc. } & =\text { locative } \\
\text { Mong. } & =\text { Mongolian } \\
\text { Osm. } & =\text { Osmanl } \\
\text { Pers. } & =\text { Persian } \\
\text { phr. } & =\text { phrase }(\mathrm{s})
\end{aligned}
$$




$$
\begin{aligned}
\text { pl. } & =\text { plural } \\
\text { Pol. } & =\text { Polish } \\
\text { poss. } & =\text { possessive } \\
\text { prob. } & =\text { probably }
\end{aligned}
$$

$$
\begin{aligned}
\text { Sp. } & =\text { Spanish } \\
\text { stand. } & =\text { standard } \\
\text { suff. } & =\text { suffix } \\
\text { T. } & =\text { Turkish }
\end{aligned}
$$

\section{References}

ArgAd. = Adamović M. (ed.). 2001. Das Türkische des 16. Jahrhunderts. Nach den Aufzeichnungen des Florentiners Filippo Argenti (1533). Göttingen.

ArgR. = Rocchi L. (ed.). 2007. Ricerche sulla lingua osmanl del XVI secolo. Il corpus lessicale turco del manoscritto fiorentino di Filippo Argenti (1533). Wiesbaden.

ArvAdd. = Arveiller R. 1999. Addenda au FEW XIX (Orientalia). Tübingen.

BassR. $\quad=$ Rocchi L. 2006. Esotismi nell'italiano cinquecentesco. Il corpus alloglotto dell'opera di Luigi Bassano da Zara. - Rivista Italiana di Linguistica e Dialettologia 8: 57-84.

BodrogPVCC $=$ Bodrogligeti A. 1971. The Persian vocabulary of the Codex Cumanicus . Budapest.

BVenON. = Venetiano B. 1580. Opera Nova de Vocaboli Turcheschi, \& Gregheschi (...). Venezia.

CardonaVOr. = Cardona G.R. 1969. Voci orientali in avvisi a stampa romani del '500. Lingua Nostra 30: 5-9.

CarrR. = Rocchi L. (ed.). 2011. Il dizionario turco-ottomano di Arcangelo Carradori (1650). Trieste.

ClausonED = Clauson G. 1972. An etymological dictionary of pre-thirteenth century Turkish. Oxford.

COED = The compact Oxford English dictionary. $1991^{2}$. Oxford.

DankoffArm. = Dankoff R. 1995. Armenian loanwords in Turkish. Wiesbaden.

DankoffEÇGl. $=$ Dankoff R. 1991. An Evliya Çelebi glossary. Unusual, dialectal and foreign words in the Seyahat-name. Boston.

DeiCr. = Dei B. 1984. "Cronica", a cura di R. Barducci. Firenze.

DELI = Cortelazzo M., Zolli P. 1979-1988. Dizionario Etimologico della Lingua Italiana. [vol. 1-5]. Bologna.

DernBab. = Babinger Fr. (ed.). 1923. Hans Dernschwams Tagebuch einer Reise nach Konstantinopel und Kleinasien (1553/55). München, Leipzig.

DValCard. = Cardini C. (ed.). 2001. La Porta d'Oriente. Lettere di Pietro Della Valle: Istanbul 1614. Roma.

ErenTDES = Eren H. 1999. Türk Dilinin Etimolojik Sözlüğ̈̈. Ankara.

ÈSTJa = Sevortjan Ė.V. et al. 1974-2003. Ėtimologičeskij Slovar' Tjurkskih Jazykov. [vol. 1-7]. Moskva.

FerrR. = Rocchi L. (ed.). 2012. Il "Dittionario della Lingua Turchesca" di Pietro Ferraguto (1611). Trieste.

GeorgHeff. = Heffening W. (ed.). 1942. Die türkischen Transkriptionstexte des Bartholomaeus Georgievits aus den Jahren 1544-1548. Leipzig.

GlITR. = Rocchi L. (ed.). 2016. Il glossario italo-turco contenuto in codice fiorentino del XVI secolo. - Zeitschrift für romanische Philologie 132(4): 1049-1064. 
GUngSt. = Stein H. (ed.). 1995/6, 1997. Das Türkische Sprachgut im “Tractatus de Moribus, Condictionibus et Nequicia Turcorum" (1481) des Georg von Ungarn. - [1] Archivum Ottomanicum 14: 39-78; [2 (Lautgeschichtliches)] Archivum Ottomanicum 15: 89-118.

GülensoyKBS = Gülensoy T. 2011. Türkiye Türkçesindeki Türkçe Sözcüklerin Köken Bilgisi Sözlüğ̈̈. [vol. 1-2]. Ankara.

HaimFarh. = Haim S. 1953. Farhang Moaser Persian-English dictionary. Tehran.

HarffSt. = Stumme H. 1914. Das Arabische und das Türkische bei Ritter Arnold von Harff. - (n. ed.). Festschrift für Ernst Windisch. Lepzig: 127-137.

HarsColl. = Nagy de Harsány J. 1672. Colloquia Familiaria Turcico Latina (...). Coloniae Brandeburgicae.

HarsHaz. = Hazai Gy. (ed.). 1973. Das Osmanisch-Türkische im XVII. Jahrhundert. Untersuchungen an den Transkriptionstexten von Jakab Nagy de Harsány. Budapest.

IllNém. = Németh J. (ed.). 1970. Die türkische Sprache in Ungarn im siebzehnten Jahrhundert. [Edition of the Illésházy-Codex (1668)]. Amsterdam, Budapest.

ITSprAd. = Adamović M. (ed.). 1975. Ein italienisch-türkisches Sprachbuch aus den Jahren 1525-1530. - Wiener Zeitschrift für die Kunde des Morgenlandes 67: 217-247. [A copy of $\rightarrow$ LupisON with a few variations].

JunkerAlaviW. = Junker H.F.J., Alavi B. 1965. Wörterbuch Persisch-Deutsch. Leipzig.

LettBomb. = Bombaci A. (ed.). 1939/1949. Una lettera turca in caratteri latini del dragomanno ottomano Ibrāhīm al veneziano Michele Membre (1567). - Rocznik Orientalistyczny 15: 129-144.

LubAd. = Adamović M. (ed.). 1977. Das osmanisch-türkische Sprachgut bei R. Lubenau. München.

LupisON. = Lupis P. ca.1520. Opera Nova de M. Pietro Lupis Valentiano. La qual insegna a parlare Turchesco. Ancona.

MachDisc. = Machiavelli N. 1984. Discorsi sopra la prima deca di Tito Livio. Premessa al testo e note di Giorgio Inglese. Milano.

MancT. = Mancini M. 1990. Turchismi a Roma e a Venezia. - Poli D. (ed.). Episteme. In ricordo di Giorgio Raimondo Cardona. Roma: 75-112.

MascVoc. = Mascis A. 1677. Vocabolario Toscano e Turchesco. Firenze.

MegILT. = Megiser H. 1612. Institutionum Linguae Turcicae Libri Quatuor. Lipsiae.

MegThP. = Megiser H. 1603. Thesaurus Polyglottus vel Dictionarium Multilinguae (...). Francofurti ad Moenum.

MenTratt. = Menavino G.A. 1548. Trattato de costumi et vita de Turchi. Firenze.

MinHist. = Minadoi G. 1587. Historia della guerra fra Turchi et Persiani. Roma.

MolDitt. = Molino G. 1641. Dittionario della lingua Italiana, Turchesca. Roma.

N. = Nişanyan S. 2009. Sözlerin Soyağacı. Çağdaş Türkçenin Etimolojik Sözlüğü. [ $4^{\text {th }}$ edition]. İstanbul.

NicQLivr. = de Nicolay N. 1568. Les quatre premiers livres des navigations et pérégrinations orientales. Lyon.

P. = Pomorska M. 2013. Materials for a historical dictionary of New Persian loanwords in Old Anatolian and Ottoman Turkish from the $13^{\text {th }}$ to the $16^{\text {th }}$ century. Kraków.

PalBern. = Palerne J. 1991. D’Alexandrie à Istanbul. Pérégrinations dans l'Empire Ottoman 1581-1583. [Introduction et annotations d'Yvelise Bernard]. Paris. [Palerne's manuscript dates back to 1584]. 
PalPD. = Palerne J. 1606. Peregrinations (...). Plus est adiousté un petit dictionaire en langage françois, italien, grec vulgaire, turc, moresque, ou arabesque, \& esclauon (...). Lyon. [See PalBern].

PaszkStach. = Stachowski M. 2013. Marcin Paszkowski's Polish and Turkish dictionary (1615). - Studies in Polish Linguistics 8: 45-55.

PostelInstr. = Postel G. 1575. Instruction des motz de la langue turquesque les plus communs, put as an introduction (without page numbering) to the book by the same author Des Histoires orientales et principalement des Turkes ou Turchiques (...). Paris.

PostelRepT. = Postel G. 1560. De la Republique des Turcs (...). Poitiers.

PostelTPart. = Postel G. 1560. La tierce partie des Orientales Histoires (...). Poitiers.

RambLibT. = Ramberti B. 1539. Libri tre delle cose de Turchi. Venezia.

RamNav. = Ramusio G.B. 1550. Delle Navigationi et Viaggi. Venezia.

RäsänenVW. = Räsänen M. 1969. Versuch eines etymologischen Wörterbuchs der Türksprachen. Helsinki.

RelAlb.I = Alberi E. (ed.). 1840. Relazioni degli ambasciatori veneti al Senato. [Serie 3, vol. 1]. Firenze.

RelAlb.III = Alberi E. (ed). 1855. Relazioni degli ambasciatori veneti al Senato. [Serie 3, vol. 3]. Firenze.

RelPedF. = Pedani-Fabris M.P. (ed.). 1996. Relazioni di ambasciatori veneti al Senato. Volume XIV. Costantinopoli - Relazioni inedite. Padova.

RJTMajd. = Majda T. (ed.). 1985. Rozwój języka tureckiego w XVII wieku (rękopis z 1611 r., ze zbiorów Biblioteki Uniwersyteckiej we Wrocławiu, sygn. M. 1529). Warszawa.

RycautPSt. = Rycaut P. 1668. The present state of the Ottoman Empire (...). London.

SchwSt. = Stein H. (ed.). 1987. Das türkische Sprachmaterial in Salomon Schweiggers Reisebuch (1608). - Acta Orientalia Academiae Scientiarum Hungaricae 41.2: 217-266.

SchweickOsm. $=$ Schweickard W. 2011. Osmanismen in den europäischen Sprachen. Vorüberlegungen zu einem vergleichenden historischen Wörterbuch. - Lexicographica 27: 221-239.

SchweickStrat. $=$ Schweickard W. 2011. La stratificazione cronologica dei turchismi in italiano. La Lingua Italiana. Storia, Strutture, Testi 7: 9-16.

SchweickTurk. $=$ Schweickard W. 2013. Turkisms in Italian, French and German (Ottoman period, 1300-1900). A historical and etymological dictionary. [http://www. uni-saarland.de/lehrstuhl/schweickard/turkisms.html].

SchweickTW. = Schweickard W. 2014. Türkische Wortgeschichte im Spiegel europäischer Quellen. - Zeitschrift für romanische Philologie 130.2: 815-832.

SorOtt. = Soranzo L. 1598. L'Ottomanno. Ferrara.

SpandSath. = Spandugnino Patritio Constantinopolitano Th. 1890. De la origine de li imperatori ottomani, ordini della corte, forma del guerreggiare loro, religione, rito, et costumi della natione. - Sathas C.N. (ed.). Documents inédits relatifs à l'histoire de la Grèce au moyen âge. [vol. 9]. Paris: 133-261.

StachSHET. = Stachowski S. 2014. Słownik historyczno-etymologiczny turcyzmów w języku polskim. Kraków.

StachWMong. = Stachowski M. 2012. Written Mongolian čamča 'shırt' and its etymological counterparts in Europe. - Hyytiäinen T. et al. (eds.). Per Urales ad Orientem. Iter polyphonicum multilingue. Helsinki: 445-451. 
StanWSPA = Stanisławski J. 1994. Wielki słownik polsko-angielski / The great PolishEnglish dictionary. Warszawa.

Steingass = Steingass F. 1892. A comprehensive Persian-English dictionary. London.

TETTL= Tietze A. 2002, 2009. Tarihi ve Etimolojik Türkiye Türkçesi Lugatı/Sprachgeschichtliches und etymologisches Wörterbuch des Türkei-Türkischen. [vol. 1: A-E]. İstanbul,Wien; [vol. 2: F-J]. Wien.

TietzePAbl. = Tietze A. 1964. Persische Ableitungssuffixe im Azerosmanischen. - Wiener Zeitschrift für die Kunde des Morgenlandes 59-60: 154-200.

TLIO = (n. ed.). 1997-. Tesoro della Lingua Italiana delle Origini. [http://www.tlio. ovi.cnr.it/TLIO/].

TMEN = Doerfer G. 1963-1975. Türkische und mongolische Elemente im Neupersischen. [vol. 1-4]. Wiesbaden.

TS = (n. ed.). 1963-1977. XIII. yüzyıldan beri Türkiye Türkçesiyle yazılmış kitaplardan toplanan tanıklariyle tarama sözlüğ̈̈. [vol. 1-8]. Ankara.

UrbanTTEt. = Urban M. 2015. The treatment of Turkic etymologies in English lexicography. Lexemes pertaining to material culture. Kraków.

VNAd. = Adamović M. (ed.). 1976. Vocabulario nuovo mit seinem türkischen Teil. Rocznik Orientalistyczny 38: 43-69.

WennStach. = Stachowski M. (ed.). 2015. Osmanisch-Türkische Appellativa im Reisebuch von Adam Wenner (1622). - Ragagnin E., Wilkens J. (eds.). Kutadgu Nom Bitig. Festschrift für Jens Peter Laut zum 60. Geburtstag. Wiesbaden: 593-607. 
